\title{
Herramientas virtuales para la enseñanza de la Diversidad Vegetal en la Educación Superior
}

\section{Virtual tools for teaching Plant Diversity in Higher Education}

ABDIEL JOVER CAPOTE ${ }^{1}$,
ORCID: 0000-0002-2040-6820, ajover@uo.edu.cu

ASIEL CABrera Guerrero ${ }^{1}$

ORCID: 0000-0002-8858-0331, asiel.cabrera@uo.edu.cu

RAMÓN SALAZAR QUEVEDO ${ }^{1}$

ORCID: 0000-0002-5597-2021, rsalazarq@uo.edu.cu

LIANNE BARRERA ROCA ${ }^{1}$

ORCID: 0000-0002-6995-1117, liannec@uo.edu.cu

${ }^{1}$ Departamento de Biología y Geografía, Facultad de Ciencias Naturales y Exactas, Universidad de Oriente. Santiago de Cuba, Cuba.

Recibido: 1을 de noviembre de 2019 - Aceptado: 1으 de mayo de 2021

\section{Resumen}

La integración de las Tecnologías de la Información y las Comunicaciones (TIC) en el proceso de formación mejora el aprendizaje significativo de los estudiantes. En el presente trabajo se valora el uso aplicación de las TIC en el proceso de enseñanza-aprendizaje de las asignaturas de sistemática vegetal en los estudiantes del primer y segundo año de la carrera de Licenciado en Biología. Los materiales se confeccionaron con la herramienta eXeLearning y Moodle en el Entorno Virtual de Aprendizaje. La aplicación de la técnica de ladov sirvió para determinar los índices de satisfacción grupal e individual de los estudiantes en relación con los recursos didácticos desarrollados. El diagnóstico inicial arrojó que los estudiantes del primer y segundo año en el curso académico 2017-2018 no emplean métodos de aprendizaje autónomo, dedican poco tiempo al estudio y solo lo hacen cuando tienen algún examen. El índice de satisfacción grupal resultó satisfactorio para las aulas virtuales y guías de estudio, mientras que en el individual prevaleció el de clara satisfacción.

Palabras clave: Guías de Estudio, Entorno Virtual de Aprendizaje, autoaprendizaje, Educación Superior.

\section{Abstract:}

The integration of Information and Communication Technologies (ICT) in the training process improves the meaningful learning of students. In the present work, the use of ICTs in the teaching-learning process of the subjects of vegetal systematics in the students of the first and second year of the Biology career is evaluated. The materials were made with the eXeLearning tool and Moodle in the Virtual Learning Environment. The application of the ladov technique served to determine the group and individual satisfaction indexes of the students about the teaching resources developed. The initial diagnosis showed that the students of the first and second year of the Bachelor's degree in Biology in the academic year 2017-2018 do not use autonomous learning methods, they spend little time studying and only do so when they have an exam. The group satisfaction index remained in the satisfactory category for virtual classrooms and study guides, while that of clear satisfaction prevailed in the individual one.

Keywords: Study Guides, Virtual Learning Environment, self-learning, Higher Education. 


\section{Introducción}

Lo tradicional y común en las aulas universitarias es que el profesor sea el único portador y responsable del conocimiento y los estudiantes tienen un desempeño pasivo en el proceso de enseñanza (Romero y Crisol, 2012). El proceso de formación continua del sujeto, idea rectora en la nueva generación de planes de estudio en Cuba conocidos como Plan E, demanda de un profesor universitario que fomente el aprendizaje creativo y autónomo haciendo que el estudiante piense por sí mismo (Crisol, 2009). Sin embargo, las nuevas cohortes de estudiantes tienen nuevas costumbres, formas de vida, ideas e incluso mentalidades que no corresponden con los modos de hacer del profesorado (Fabra y Doménech, 2001; Masjuan, 2004).

Las Tecnologías de la Información y las Comunicaciones constituyen una valiosa herramienta para ser utilizada en la enseñanza que contribuye a que el proceso de enseñanza y de aprendizaje esté más enfocado en el aprendizaje del estudiante que en la enseñanza del profesor (Goñi, 2005). Dentro de las posibilidades de las TIC encontramos los Entornos Virtuales de Aprendizajes (EVA) los cuales proporcionan herramientas de aprendizaje más estimulantes y motivadoras, a partir de las cuales el estudiante se implica en la solución de un problema, con un medio interactivo, que le permite reflexionar, intercambiar criterios y acceder a la información, desde cualquier lugar, sin limitaciones, con la posibilidad de ampliar el universo del saber y enriquecer su cultura (Ulloa y Velázquez, 2009).

El aprendizaje de los contenidos referidos a la diversidad vegetal en la carrera de Biología requiere no solo la memorización de conceptos, estructuras y características de los grupos taxonómicos, sino que demanda el desarrollo y la estimulación de la actividad reflexiva de los estudiantes. De esta manera se puede relacionar la teoría con la práctica y solucionar problemas desde sus propias estrategias para la construcción de un conocimiento enriquecido (Velázquez, 2005; Guerra, Campo y Palomino, 2017). Hoy, las herramientas tecnológicas y las estrategias de aprendizaje que pueden proponerse apartir de ellas, exigen que el estudiante adopte un rol activo e interactivo en suproceso de formación (Salinas, 2011).

Las TIC permiten que los estudiantesde manera activa puedan disponer de materiales actualizados y guías de estudios autónomas de manera que identifiquen sus necesidades formativas, establezcan sus objetivos de aprendizaje y eleven su motivación al inicio y durante el itinerario de aprendizaje y generen confianza en sí mismo (Romero y Crisol, 2012). Existen muchas herramientas que permiten el trabajo autónomo, pero el instrumento didáctico más relevante y sistemático en este sentido es la guía de estudio autónomo (Salvador y Gallego, 2009). Estas guías se consideran un elemento vertebrador del aprendizaje por descubrimiento, dado que en ella se articulan técnicas de trabajo intelectual y de investigación, actividades tanto individuales como grupales, experiencias curriculares y extracurriculares (Romero y Crisol, 2009).

Las asignaturas Biología de Algas y Hongos (1er año) y Sistemática de Plantas I y II (2do año), constituyen las tres asignaturas que contienen los contenidos de sistemática y diversidad vegetal en la carrera de Biología (Ministerio de Educación Superior [MES], 2007). Es característico que los estudiantes vean el estudio de estas asignaturas a través de recursos de memorización lo que limita la motivación y el aprendizaje de las mismas (Silva, Pinho, López, Nogueira y Silveira, 2010; Cabrera y Jover, 2017).

En diagnósticos realizados a estudiantes que cursan estas asignaturas se han constatados dificultades como: pobre nivel de desarrollo logrado por los estudiantes en el aprendizaje, limitaciones con respecto al esfuerzo que realizan para pensar y limitaciones con el uso de diferentes recursos informáticos durante el proceso de aprendizaje. Por tanto, se aprecian insuficiencias cognitivas e instrumentales en el proceso de aprendizaje de los contenidos de sistemática vegetal, que limitan el desempeño profesional de los estudiantes.

El presente trabajo tiene como objetivo evaluar la satisfacción de las Aulas Virtuales en la enseñanza de la Diversidad Vegetal dirigidas a mejorar las estrategias de aprendizaje autónomo en los estudiantes matriculados en el primer y el segundo año de carrera de Biología en la Universidad de Oriente de Cuba.

\section{Fundamentos teóricos}

El modelo del aprendizaje autónomo permite al individuo ser autor de su propio desarrollo, eligiendo los caminos, las estrategias, las herramientas y los momentos que considere pertinentes para aprender y poner en práctica, de manera independiente, lo que ha aprendido (González, Garduño y Puga, 2017). En este modelo los estudiantes son los principales agentes del desarrollo del aprendizaje (Porras-Velásquez, 2010). Además, en este tipo de aprendizaje promueve una metodología que aproveche las experiencias que 
el sujeto ha adquirido en su vida (Escribano, 1995). Por su parte el profesor pasa de ser un expositor e instructor por excelencia a convertirse en un tutor o facilitador del proceso de aprendizaje utilizando diferentes metodologías para movilizar los conocimientos producidos por los alumnos (Aburto, 2020). Además, los profesores se deben caracterizar por promover en los estudiantes la motivación por la materia y el deseo de aprender por si mismos (Ramsden, 2002).

Los entornos virtuales, como herramienta tecnológica, facilitan la interacción y promueven el aprendizaje autónomo a partir de las interacciones que se logran, del tipo alumnos-contenido, alumno-alumno y alumno-profesor (Rodríguez y Espinoza, 2017). Estos entornos facilitan la divulgación de contenidos, desarrollan habilidades, propician espacios de evaluación y de gestión del conocimiento en los diferentes modelos educativos (Rodríguez y Barragán, 2017). Es importante señalar que los entornos virtuales de aprendizaje potencian el papel protagónico del sujeto que aprende y favorece la mediación del profesor-tutor a través de las herramientas de gestión de recursos y las posibilidades de comunicación sincrónica y asincrónica que ofrece este recurso (Morado y Ocampo, 2019). Estas herramientas tecnológicas generan es- pacios para la autoevaluación de los estudiantes pues permiten crear preguntas, configurar ejercicios y corrección de las respuestas de una forma automática (Beltrán, 2016). Sin embargo, para lograr el éxito de estos entornos virtuales en el proceso de enseñanza y de aprendizaje es necesario una planificación cautelosa de las actividades y recursos educativos basada en las necesidades y características de los alumnos (Cedeño y Muriño, 2019).

Moodle (Modular Object-Oriented Dynamic Learning Enviroment) o Entorno Modular Dinámico Orientado a Objetos de Aprendizaje es un software para la creación de cursos y sitios web basados en Internet con el propósito de que el conocimiento se va construyendo por el estudiante a partir de su participación activa en el proceso y en relación con su entorno social (Casales, Rojas y Paulí, 2008). Dentro de las opciones más atractivos de esta plataforma esta la creación de glosarios, lecciones, orientaciones docentes, actividades de aprendizaje y el vínculo a otros recursos de internet (Torres-Diaz, Jara y Valdiviezo, 2013). Esta plataforma permite la integración de cualquier recurso digital que puede ser reutilizado para apoyar el aprendizaje utilizando paquetes SCORM (Mínguez y Diago, 2013). Una herramienta de fácil utilización por los profesores en

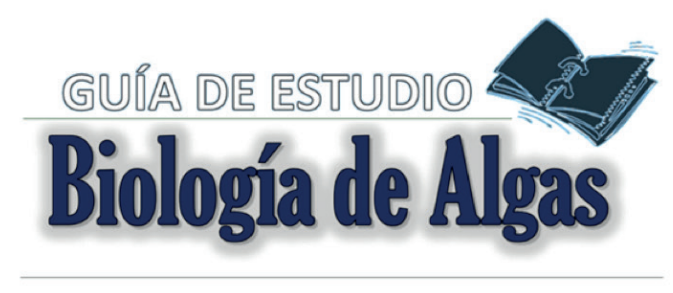

Abdiel Jover Capote y Asiel Cabrera Guerrero DEPARTAMENTO DE BIOLOGÍA Y GEOGRAFÍA | UNIVERSIDAD DE ORIENTE

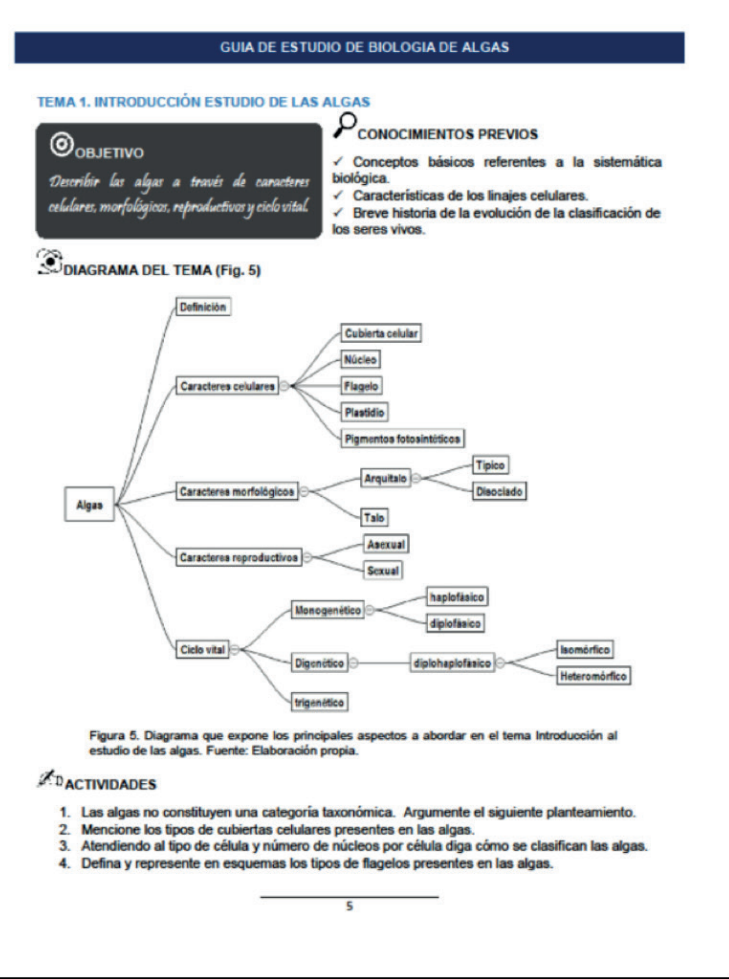

Fig. 1. Ejemplo de las Guías de Estudio Autónomo elaboradas para las asignaturas de Diversidad Vegetal en la Universidad de Oriente, Cuba 
la elaboración de paquetes SCORM es ExeLearnig ( $\mathrm{Ca}$ rranza, Hernández y Hernández, 2013).

La acción del profesor en el modelo de aprendizaje autónomo se concreta a través de las guías de estudio (García y de la Cruz, 2004). En la literatura pedagógica se registran diferentes nombres para identificar a este recurso didáctico como son: guías didácticas, guías de estudio autónomo o guía docente (Aguilar, 2004). Según esta autora, estas guías promueven el aprendizaje autónomo al aproximar el material de estudio al alumno (texto convencional y otras fuentes de información), a través de diversos recursos didácticos (explicaciones, ejemplos, comentarios, esquemas y otras acciones similares a la que realiza el profesor en clase). En ellas se relacionan técnicas de trabajo intelectual, de investigación, actividades tanto individuales como grupales y experiencias curriculares y extracurriculares (Romero y Crisol, 2009). Según Bruer (1995) estas guías se convierten en el "andamiaje" que posibilita al estudiante avanzar con mayor seguridad en el aprendizaje autónomo. Por lo que se puede resumir que las guías didácticas tienen como principal objetivo lograr concretar el papel orientador del docente y consolidar la actividad independiente del alumno a través de la tarea docente como célula básica del proceso docente educativo (García y de la Cruz, 2004).

\section{Materiales y métodos}

Se desarrolló un estudio experimental en el que participaron los estudiantes del primer y segundo año de la carrera de Biología, 22 y 14 respectivamente, perteneciente a la Facultad de Ciencias Naturales y Exactas de la Universidad de Oriente. Se escogieron la totalidad de los estudiantes del primer y segundo año, pues las matrículas son pequeñas. Todos dieron su consentimiento para participar en la investigación. El periodo de aplicación y evaluación de la investigación fue el primer semestre del curso académico 2017-2018.

La investigación se desarrolló en cuatro etapas, en la primera etapa para realizar el diagnóstico se aplicó un cuestionario al inicio de las asignaturas para obtener la información referida a las estrategias de aprendizaje de los estudiantes, motivación para el aprendizaje, organización de las actividades de aprendizaje y técnicas

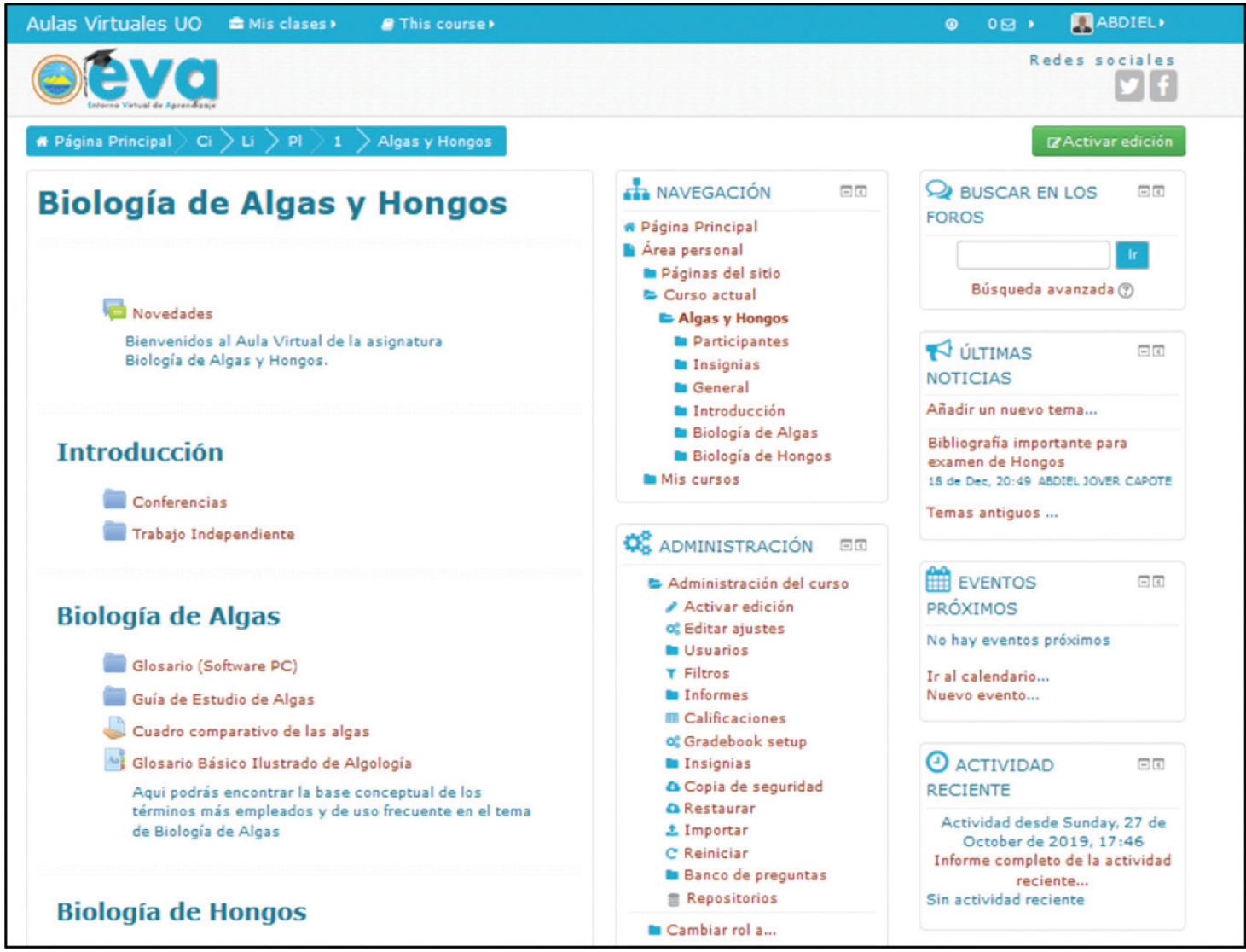

Fig. 2. Aula Virtual de la asignatura Biología de Algas y Hongos en el Entorno Virtual de Aprendizaje de la Universidad de Oriente 


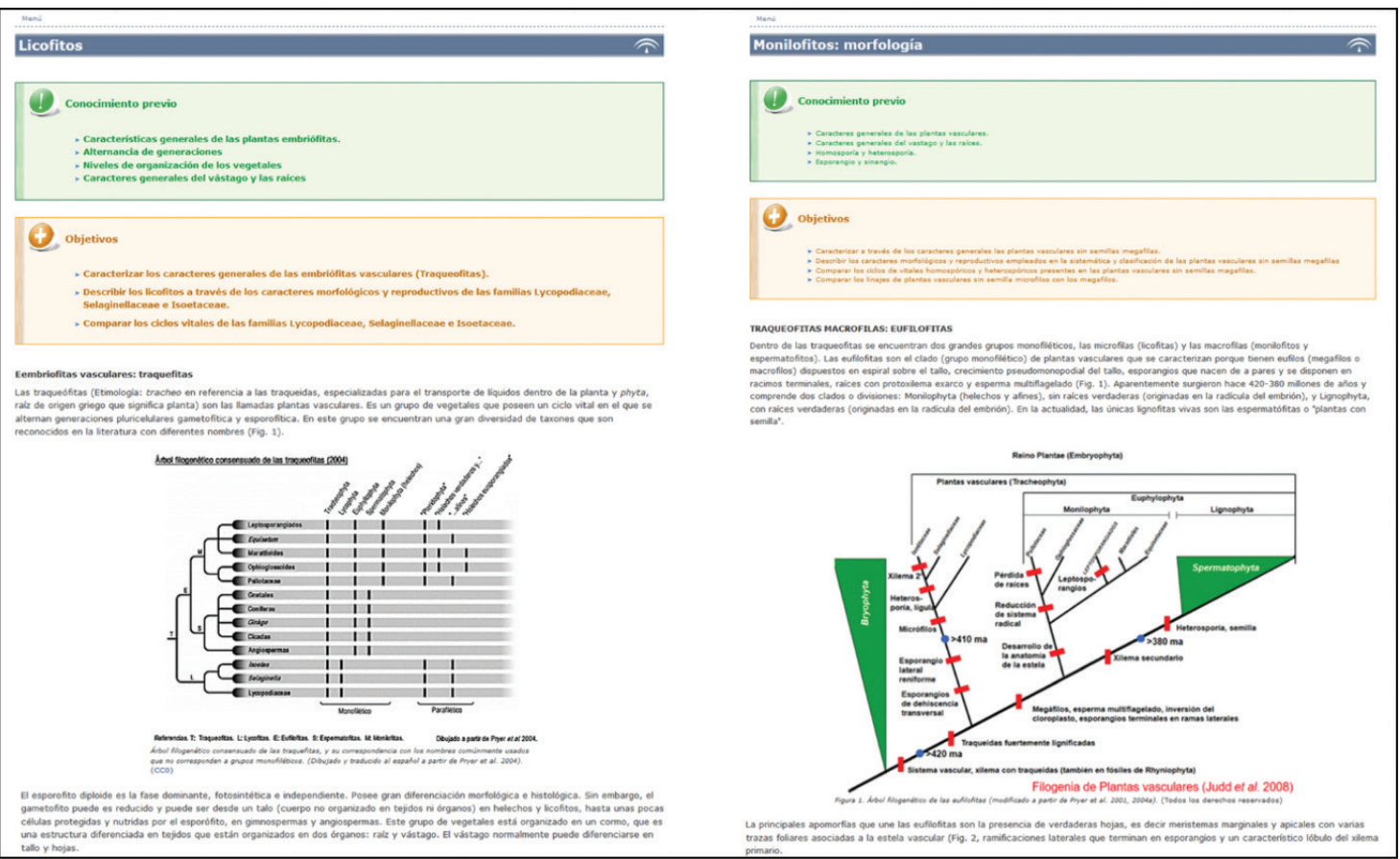

Fig. 3. Guías de Aprendizaje para la enseñanza de la Diversidad Vegetal creadas en el software libre Exelearning disponibles en el Entorno Virtual de Aprendizaje de la Universidad de Oriente

para la auto preparación. El mismo se realizó a través de un cuestionario que se les aplicó a todos los estudiantes que participaron de la investigación (Anexo 1). Los datos fueron procesados, sistematizados y obtenidas las regularidades.

En la segunda etapa se realizó la compilación y análisis de la información para la confección de las Guías de Estudio Autónomas (Figs. 1, 2 y 3) y los Glosarios de Términos asociadas en las mismas. En el desarrollo de esta etapa se utilizaron métodos teóricos como el análisis y la síntesis la deducción e inducción. Estos fueron necesarios en la determinación de los fundamentos teóricos y metodológicos relacionados con el aprendizaje autónomo a través del aula virtual.

En esta etapa se revisó la literatura básica (Recio y Maldonado, 2013; Judd, Campbell, Kellogg, Stevens y Donoghue, 2016) y complementaria (Izco, 2004; Bresinsky, Koörner, Kadereit, Neuhaus y Sonnewald, 2013; Raven, Evert y Eichhorn, 2013; Simpsom, 2019) de las asignaturas de Sistemática Vegetal. En esta búsqueda se sistematizaron y profundizaron los contenidos y orientaciones metodológicas establecidos en el Plan de Estudio por la Comisión Nacional de la Carrera de Biología (MES, 2007). A partir de esta información se seleccionaron los ejemplos, explicaciones, conceptos, actualización sistemática y ejercicios paraconfeccionar las tres guías de estudio autónomas y los dos glosarios de términos.
En la producción de los materiales didácticos se identificaron como elementos de entrada la gestión tecnológica (valoración de las diferentes herramientas informáticas para la confección), gestión académica (libros de textos, información de la carpeta metodológica de la asignatura, búsqueda de las experiencias de enseñanza en otras universidades) y el proceso de innovación educativa (generación de ejercicios, síntesis y desarrollos de contenidos) (Brito y Rivero, 2014).

El soporte que se seleccionó es el eXeLearning, pues es un programa libre y abierto bajo licencia GPL-2. Los recursos creados en exelearning son accesibles en formato XHTML o HTML5, pudiendo generarse sitios web completos (páginas web navegables), insertar contenidos interactivos (preguntas y actividades de diferentes tipos) en cada página, exportar los contenidos creados en otros formatos como ePub3 (un estándar abierto para libros electrónicos), IMS o SCORM (estándares educativos que permiten incorporar los contenidos en herramientas como Moodle), XLIFF (un estándar para la traducción) y catalogar los contenidos con diferentes modelos de metadatos: Dublin Core, LOM, LOM-ES. Los materiales se incorporan en el Entorno Virtual de Aprendizaje de las asignaturas disponible en: www.eva.uo.edu.cu.

Las herramientas didácticas colocadas en la plataforma Moodle fueron evaluadasatendiendo a la satisfac- 
ción de la población estudiada, utilizando la técnica de ladov (Fernández y López, 2014; Roque, Valdivia, Zalagaz y Alonso, 2017). El instrumento para determinar la satisfacción contó con 18 preguntas, cuya relación es ignorada por los estudiantes (Anexo 2). El resultado interrelacionado de las preguntas 1, 2 y 7 indicaron la satisfacción individual con las aulas virtuales y las preguntas 10, 14 y 16 con las guías de estudio autónomas. En la evaluación se siguió la siguiente escala:

1. Clara satisfacción.

2. Más satisfecho que insatisfecho.

3. No definida.

4. Más insatisfecho que satisfecho.

5. Clara insatisfacción.

Esta técnica también permite obtener el índice de satisfacción grupal (ISG), expresado en una escala numérica que oscila entre +1 y -1 , dada por la siguiente ecuación:

$$
I S G=\lfloor A(+1)+B(+0,5)+C(0)+D(-0,5)+E(-1) / N\rfloor
$$

Donde: A, B, C, D, E, representan el número de sujetos en cada categoría definida para la satisfacción individual; y $\mathrm{N}$ representa el número total de individuos del grupo. El índice grupal puede arrojar valores entre +1 y -1. La insatisfacción está dada por valores entre -1 y $-0,5$, contradicción entre $-0,49$ y $+0,49$ y la satisfacción entre 0,5 y 1 .

\section{Resultados y discusión}

El diagnóstico inicial arrojó que el $81 \%$ de los estudiantes del primer y segundo año de la carrera de Biología en el curso académico 2017-2018 les gusta el estudio y el $78 \%$ utiliza al menos una técnica de estudio. Las técnicas de estudio que emplean con mayor frecuencia son la repetición, la toma de apuntes y la elaboración de resúmenes (Fig. 4).

A la hora de realizar el estudio el $75 \%$ mostraron preferencias por espacios en el hogar y el $44 \%$ y $33 \%$ lo realiza con sus amistades y con los estudiantes sin problemas docentes respectivamente. Otra característica que distinguen a estos estudiantes es que son finalista, el $78 \%$ estudia antes de las evaluaciones, mientras que el resto lo realiza semanalmente. También, dedican poco tiempo para estudiar, el $56 \%$ dedica menos de una hora y los demás entre una y tres horas. Además, solo emplean como fuentes en el estudio internet $31 \%$ y el $69 \%$ las notas de clase.

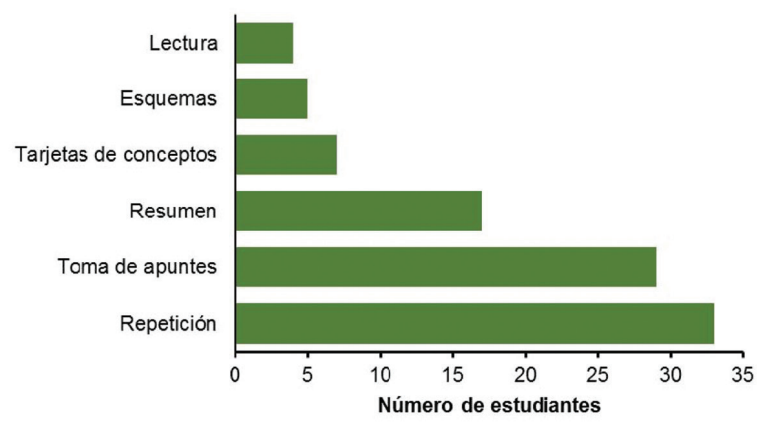

Fig. 4. Técnicas de estudios más usadas por los estudiantes del primer y segundo año de la carrera de Biología en la Universidad de Oriente de Cuba durante el curso académico 2017-2018.

Estas características presentes en los estudiantes del primer y segundo año se ven reflejadas en sus resultados docentes en las asignaturas de sistemática y diversidad vegetal. En donde más del $60 \%$ de los estudiantes desaprueban las evaluaciones frecuentes y exámenes realizados en las primeras seis semanas del curso. Aunque los factores que inciden en el rendimiento académico de los estudiantes universitarios poseen una multidimensionalidad caracterizada en factores sociales, personales, institucionales, tecnológicas y académicas; según Navarro y Blandón (2017) las estrategias de aprendizaje, el tiempo y la dedicación al estudio son esenciales. Además, se ha encontrado que las estrategias de aprendizaje con una correlación significativa con el promedio de calificaciones de los estudiantes universitarios son: elaboración, revisión, constancia, pensamiento crítico, uso del tiempo y concentración (Sotelo, Echeverría, Ramos y Barrera, 2015). Sin embargo, los profesores pueden jugar un papel activo en proceso formativo al intervenir en la dedicación al estudio desde la implementación de estrategias que permitan al estudiante mantenerse centrado en los estudios (Artola, Rodríguez y Reyes, 2020).

A partir de este diagnóstico se realizaron tres guías de estudio autónomo para las asignaturas: Biología de las Algas, Biología de los Hongos y Biología de las Embriofitas.

En las mismas se definen los objetivos de estudio, los conocimientos previos que deben tener los estudiantes, las actividades, preguntas de conexión evolutiva, glosario y bibliografía recomendada. Las preguntas sobre evolución son importantes por servir de enlace 
e integrar el contenido de los diferentes temas de las asignaturas. Estas guías didácticas constituyen un recurso que tiene el propósito de orientar metodológicamente al estudiante en su actividad independiente, al mismo tiempo que sirven de apoyo a la dinámica del proceso docente, guiando al alumno en su aprendizaje, favorecen este proceso y promueven la autonomía a través de diferentes recursos didácticos como son: explicaciones, ejemplos, comentarios, esquemas, gráficos y mapas mentales y conceptuales (García y de la Cruz, 2014).

Estos materiales junto a otros complementarios se colocaron en las Aulas Virtuales creadas en Moodle en el Entorno Virtual de Aprendizaje de la Universidad de Oriente (Fig.2) de manera que estuvo disponible para todos los estudiantes. Estas herramientas virtuales son esenciales para la organización y desarrollo de la actividad del profesor y del estudiante en la docencia y fuera de ella, especialmente en lo concerniente al uso de las tareas incluidas en ellas como metodología de trabajo para los estudiantes (López-Zafra, RodríguezEspartal, Martínez y Landa, 2015).

Las guías de estudios se montaron en soporte digital utilizando las potencialidades que brinda el software libre eXelearning.

Esta herramienta informática tiene ventajas formidables para el aprendizaje. Además, se pueden hacer aportaciones para enriquecer el contenido y se solucionan problemas de forma interactiva sobre la base de una navegación sencilla e intuitiva a partir de elementos simples como documentos con textos, actividades de autoevaluación, hipervínculos, imágenes y otras (Hernández, Valenciano, Serrano, Bles y Coca, 2018).

Al finalizar las asignaturas el $83 \%$ utilizó durante el estudio las guías de estudio. Las secciones de la guía glosario, las actividades y el diagrama del tema fueron las que más les gustaron a los estudiantes en un $92 \%, 75 \%$ y $53 \%$ respectivamente. Sin embargo, las secciones que implicaban integrar conocimiento entre los temas de las asignaturas fueron las que menos le gustaron, la sección conexión evolutiva (97\%) y conocimientos previos (83\%). La importancia del uso de las guías de estudio en las asignaturas de Sistemática Vegetal, según el criterio de los encuestados, radican en que orientan como apropiarse de los contenidos de las asignaturas, permiten sistematizar elementos a tratar en cada tema y propician la construcción de esquemas, cuadros y otras representaciones gráficas en la que se resumen los contenidos. Por último, la principal limitación que detectan son las relacionadas con la presencia de una sola introducción general y que solo se pone la bibliografía general y no se desglosa por tema.

En el proceso de aprendizaje los estudiantes encuentran en las guías de estudios un medio que les facilita la comprensión del contenido (Aguilar, 2004). Según esta autora, las guías proponen metas claras que orientan el estudio de los alumnos, sugieren técnicas de trabajo intelectual que faciliten la comprensión del texto y contribuyan a un estudio eficaz y sugieren distintas actividades y ejercicios, en un esfuerzo por atender los distintos estilos de aprendizaje. Las secciones de mayor preferencia fueron las de orientación y las que les permitieron realizar el estudio independiente en las asignaturas de diversidad vegetal. Este resultado coincide con el de Mateo (2013) quien plantea que las guías de estudio generan una sinergia en el aprendizaje autónomo y en el estudio independiente para los alumnos. Un reto de los docentes es profundizar en el tratamiento de la integración de los contenidos desde la clase, de manera que los estudiantes puedan aprovechar las ventajas que tiene la sección conexiones evolutivas en las guías de estudio. Esta sección los entrena para que reflexionen sobre sus propios aprendizajes y les permite integrar en sus análisis una serie de elementos que le faciliten luego recordar sus conocimientos (Avilés, 2011).

Al concluir las asignaturas de Sistemática Vegetal en la carrera de Biología de la Universidad de Oriente 89\% de los estudiantes de los dos rimeros años habían utilizado las Aulas Virtuales en el estudio. Las herramientas de las Aulas Virtuales que más les gustaron fueron las tareas, los paquetes SCORM eXelearning que contenían las guías de estudio y los glosarios en un $89 \%$, $78 \%$ y $72 \%$ de preferencia respectivamente. Sin embargo, las que mostraron menos preferencias fueron las actividades y las carpetas en un $75 \%$ y $67 \%$ respectivamente. A partir del criterio de los estudiantes, ellos identifican que el uso de las Aulas Virtuales de Sistemática Vegetal les da acceso a los recursos didácticos en cualquier momento, les permite una constante retroalimentación con los profesores y potencia la interacción estudiante-profesor más allá del espacio de la clase. Sin embargo, las principales limitantes en el uso de las misma radican en las dificultades de conectividad y en la poca disponibilidad de computadoras.

El uso de los Entornos Virtuales de Aprendizaje radica que es una herramienta de apoyo a la docencia presencial, semipresencial y a distancia. Esto es posible porque pueden ser aplicables en diferentes contextos y con distintas actividades, ya que originan un ambien- 
te lúdico en su proceso de aprendizaje, impulsan la capacidad de superación, promueven la motivación y mejoran la creatividad (Ortiz, Torres y Cuevas, 2013). El reto del profesor universitario radica en aprovechar las ventajas que brindan las aulas virtuales y desde la instrucción y la práctica docente prevenir las desventajas (Quiroga, Jaramillo y Vanegas, 2019). Estos espacios virtuales desarrollan estilos de aprendizaje en los estudiantes, pero para lograr un mayor impacto es importante fortalecer en el espacio presencial y virtual la interacción con los estudiantes y el proceso de retro alimentación de los contenidos (Cedeño y Muriño, 2019). En general el uso de las herramientas que brindan las Aulas Virtuales de Sistemática Vegetal propicia actividades y módulos que potencian el aprendizaje autónomo. Sin embargo, las actividades, contenidos y propuestas didácticas deben ser validadas a través del trabajo metodológico de los profesores. Este elemento es importante porque el producto final esperado debe ser resultado de un proceso, por lo que se deben diseñar actividades de aprendizaje que, por etapas, contribuyan a obtener ese resultado final esperado, sin dejar de controlar y retroalimentar al estudiante (García, et al 2015).

El 82,5\% de los estudiantes del primer y el segundo año de la carrera de Biología en la Universidad de Oriente, de manera individual, muestra satisfacción con el uso de las Guías de Estudio y el $80 \%$ con las Aulas Virtuales de las asignaturas de sistemática vegetal (Tabla 1). Por su parte el Índice de Satisfacción Grupal fue de 0,71 para las Guías de Estudio y de 0,66 para el Aula Virtual. El valor del Índice es alto, lo que refleja aceptación de las herramientas virtuales en la enseñanza de la diversidad vegetal por su utilidad como herramienta para el desarrollo del aprendizaje autónomo.

Tabla 1. Relación de la satisfacción individual con la escala de satisfacción con las Guías de Estudio y las Aulas Virtuales de Sistemática Vegetal.

\begin{tabular}{|c|c|c|c|c|c|}
\multirow{2}{*}{ Escala } & \multirow{2}{*}{ Significado } & \multicolumn{2}{|c|}{ Guías de Estudio } & \multicolumn{2}{c|}{ Aula Virtual } \\
\cline { 3 - 6 } & & Satisfacción Individual & $\%$ & Satisfacción Individual & $\%$ \\
\hline+1 & Clara satisfacción. & 26 & 65 & 20 & 50 \\
\hline$+0,5$ & Más satisfecho que insatisfecho & 7 & 17,5 & 12 & 30 \\
\hline 0 & No definida & 1 & 2,5 & 3 & 7,5 \\
\hline$-0,5$ & Más insatisfecho que satisfecho & 2 & 5 & 1 & 2,5 \\
\hline-1 & Clara insatisfacción & - & - & - & - \\
\hline
\end{tabular}

\section{Conclusiones}

Las herramientas virtuales diseñadas para las asignaturas de Sistemática Vegetal en la carrera de Biología de la Universidad de Orienta y depositadas en el Entorno Virtual de Aprendizaje contribuyen al aprendizaje autónomo de los estudiantes de los dos primeros años de esta carrera. Las guías didácticas, como mediadoras del aprendizaje, tienen la potencialidad de incluir estrategias para el desarrollo de la autonomía del estudiante en las orientaciones para el estudio. Los estudiantes muestran elevada satisfacción de manera individual y colectiva con las herramientas virtuales en las asignaturas de sistemática vegetal por presentar orientación metodológica, información del contenido, indicadores de logros de los estudiantes y permiten la autoevaluación desarrollando un aprendizaje propio.

\section{Agradecimientos}

A los estudiantes del primer y segundo año de la carrera de Biología en la universidad de Oriente que brindaron su disposición y cooperación en el desarrollo de la investigación. A la profesora Eumelia Romero Pacheco y Eufemia Figueroa Corrales por los comentarios y sugerencias. A los revisores que brindaron sugerencias que permitieron la mejorar el manuscrito. 


\section{Referencias bibliográficas}

Abuto, P. (2020). El rol del profesor universitario en el siglo XXI, ¿es necesario de cambios en su actuación como docente-tutor-investigador? Revista Compromiso Social, 3: 59-72. https:// revistacompromisosocial.unan.edu.ni/index.php/ CompromisoSocial/article/view/66

Aguilar, R. (2004). La guía didáctica, un material educativo para promover el aprendizaje autónomo. Evaluación y mejoramiento de su calidad en la modalidad abierta y a distancia de la UTPL. RIED: revista iberoamericana de educación a distancia, 7(1-2): 179-192. http://e-spacio.uned. es/fez/view/bibliuned:20639

Artola, A. E., Rodríguez, C. M. M., y Reyes, C. E. M. (2020). Dedicación al estudio en jóvenes universitarios: responsabilidad compartida entre docentes y estudiantes. REFCaIE: Revista Electrónica Formación y Calidad Educativa, 8(2): 234-247. http://www.refcale.uleam.edu.ec/index. php/refcale/article/view/3237

Avilés, G. (2011). La metodología indagatoria: una mirada hacia el aprendizaje significativo desde" Charpack y Vygotsky". Intersedes: Revista de las sedes regionales, 12(23): 133-144. https:// revistas.ucr.ac.cr/index.php/intersedes/article/ view/981

Beltrán, Á. G. (2016). La autoevaluación como actividad docente en entornos virtuales de aprendizaje. Revista de Educación a Distancia, 11:1-14. https:// revistas.um.es/red/article/view/272241

Bresinsky, A., Koörner C., Kadereit J., Neuhaus G., y Sonnewald U. (2013). Strasburger's Plant Sciences. Berlín, Alemania: Springer.

Brito, J., y Rivero, M. (2014). El proceso de producción en la construcción de ambientes virtuales de enseñanza, aprendizaje y comunicación: indicadores para evaluar su calidad. Virtualidad, Educación y Ciencia, 5(8): 18-28. https://revistas. unc.edu.ar/index.php/vesc/article/view/7471

Bruer, J. (1995). Escuelas para pensar, una ciencia de aprendizaje en el aula. Madrid, España: Paidós.

Cabrera, A., y Jover, A. (2017). Valor didáctico del uso de claves interactivas en la asignatura Biología de Algas y Hongos. Revista Cubana de Ciencias Biológicas, 5(3): 1-10. http://www.rccb.uh.cu/ index.php/RCCB/article/view/185
Carranza, C., Hernández Y., y Hernández C. 2013. El eXeLearning como herramienta para la construcción de objetos de aprendizaje. Revista Material Didáctico Innovador, 9(1): 9-18. http:// www.revistamdi.uam.mx/

Casales, R., Rojas, J., y Paulí, G. (2008). Algunas experiencias didácticas en el entorno de la plataforma Moodle. Revista de informática educativa y medios audiovisuales, 5(10): 1-10. http://laboratorios. fi.uba.ar/lie/Revista/articulos.htm

Cedeño, E., y Muriño J. (2019). Entornos virtuales de aprendizaje y su rol innovador en el proceso de enseñanza. ReHuSo: Revista de Ciencias Humanísticas y Sociales, 4(1): 119-127, DOI: 10.33936/rehuso.v4i1.2156.

Crisol, E. (2009). Participación, opinión y expectativas de los estudiantes en el Espacio Europeo de Educación Superior. Granada, España: Universidad de Granada.

Escribano, A. (1995). Aprendizaje cooperativo y autónomo en la enseñanza universitaria. Enseñanza, 13: 89-102. https://gredos.usal.es/ handle/10366/69492

Fabra, M. L., y Doménech, M. (2001). Hablar y escuchar. Relatos de profesores y estudiantes. Barcelona, España: Paidós.

Fernández, F., y López. A. (2014). Validación mediante criterio de usuarios del sistema de indicadores para prever, diseñar y medir el impacto en los proyectos de investigación del sector agropecuario. Revista Ciencias Técnicas Agropecuarias, 23(3): 77-82. https://revistas.unah.edu.cu/index.php/rcta/ article/view/309

García, A., Guerrero, R. S., y Granados, J. M. (2015). Buenas prácticas en los entornos virtuales de enseñanza-aprendizaje. Revista Cubana de Educación Superior, 34(3): 76-88. http://www. rces.uh.cu/index.php/RCES/article/view/87

García, I., y de la Cruz, G. (2014). Las guías didácticas: recursos necesarios para el aprendizaje autónomo. EDUMECENTRO, 6(3): 162-175. http://www. revedumecentro.sld.cu/index.php/edumc/article/ view/378

González, Y., Garduño, M. D. L. V., y Puga, A. M. M. (2017). Estrategias que favorecen el aprendizaje autónomo en estudiantes universitarios. Caleidoscopio-Revista Semestral de Ciencias Sociales y Humanidades, (37): 75-90. DOI: 10.33064/37crscsh903. 
Goñi,J.(2005).Elespacioeuropeode Educación Superior, un reto para la universidad. Competencias, tareas y evaluación, los ejes del currículum universitario. Barcelona, España: Octaedro.

Guerra, M., Campos, L., y Palomino, M. (2017). Aulas virtuales para el aprendizaje reflexivo de la biología. Transformación, 13(3): 371-383. https:// revistas.reduc.edu.cu/index.php/transformacion/ article/view/1663

Hernández, V., Valenciano, V., Serrano, L., Bles, A., y Coca, J. (2018). Aprendizaje de las medidas de tendencia central a través de la herramienta EXeLearning. MEDISAN, 22(3): 258 . http:// scielo.sld.cu/scielo.php?script=sci_arttext\&pid =S1029-30192018000300006

Izco, J. (Ed.) (2004). Botánica. Madrid, Espala: McgrawHill/Interamericana.

Mínguez, R. T., y Diago, C. C. (2013). Revisión de herramientas de autor para el diseño de actividades educativas. DIM: Didáctica, Innovación y Multimedia, 9(25), 1-11. https://dialnet.unirioja. es/servlet/articulo?codigo $=5008759$

Judd, W.S., Campbell C.S., Kellogg E.A., Stevens P.F., y Donoghue M. J. (2016). Plant Systematics: A Phylogenetic Approach. Sunderland, USA: Sinauer Associates.

López-Zafra, E., Rodríguez-Espartal, N., Martínez, L., y Landa, J. (2015). Avaluaciód'una experiencia d'Aprenentatge Basat en Problemes (ABP) en estudiants universitaris. Revista d'Innovació Docent Universitària, 7: 71-80. https://www.raco. cat/index.php/RIDU/article/view/286964

Masjuan, J. M. (2004). Convergencia europea, reformas universitarias y prácticas de los estudiantes. EDUCAR, 33: 59-71, DOI: 10.5565/rev/educar.261.

Mateo, L. (2013). La guía didáctica: práctica de base en el proceso de enseñanza-aprendizaje y en la gestión del conocimiento. Apertura, 5(1): 66-73. http://www.udgvirtual.udg.mx/apertura/index. php/apertura/article/view/366/

Ministerio de Educación Superior [MES] (2007). Carrera de Biología: Plan de Estudio D. Facultad de Biología, Universidad de La Habana, Cuba.

Morado, M. F., y Ocampo, S. (2019). Una experiencia de acompañamiento tecno-pedagógico para la construcción de entornos virtuales de aprendizaje en educación superior. Revista Educación, 43(1): 43-61, DOI: 10.15517/revedu.v43i1.28457.
Navarro, S., y Blandón. S. (2017). Determinantes que inciden en la calidad de rendimiento académico de los estudiantes de ingeniería. Revista Científica de FAREM-Estelí, 24: 126-142, DOI: 10.5377/farem. v0i24.5556.

Ortiz, V., Torres, J., y Cuevas, R. (2013). Entornos virtuales de enseñanza y aprendizaje: la educación entre lo presencial y lo virtual. Revista Vínculos, 10(2): 435-442, DOI: 10.14483/2322939X.6567.

Porras-Velásquez, N. R. (2010). La psicología y la educación a distancia: frente al reto de aprender desde la autonomía. Poiésis, 19: 1-18, DOI: 10.21501/16920945.110

Quiroga, L. P., Jaramillo, S., y Vanegas, O. L. (2019). Ventajas y desventajas de las TIC en la educación "Desde la primera infancia hasta la educación superior". Revista Educación y Pensamiento, 26(26): 77-85. http:// www.educacionypensamiento.colegiohispano.edu. co/index.php/revistaeyp/article/view/103

Ramsden, P. (2002). Learning to teach in higher education. New York, USA: Taylor \& Francis.

Raven, P., Evert R. F., y Eichhorn S. E. (2013). Biology of Plants. New York. USA: W.H. Freeman and Company.

Recio, G., y Maldonado S. (2013). Hongos y Algas. La Habana, Cuba: Editorial Félix Varela.

Rodríguez, M., y Barragán H. (2017). Entornos virtuales de aprendizaje como apoyo a la enseñanza presencial para potenciar el proceso educativo. Killkana sociales: Revista de Investigación Científica, 1(2): 7-14. https://dialnet.unirioja.es/ servlet/articulo?codigo $=6297476$

Rodríguez, R., y Espinoza, L. A. (2017). Trabajo colaborativo y estrategias de aprendizaje en entornos virtuales en jóvenes universitarios. Revista Iberoamericana para la Investigación y el Desarrollo Educativo, 7(14): 86-109, DOI: 10.23913/ride.v7i14.274.

Romero, A., y Crisol, E. (2009). La guía de trabajo autónomo en la experiencia ECTS de la Titulación de Logopedia. En: M. Cerezo y R. Grau (Ed.), Los Nuevos Títulos de Grado: Retos y Oportunidades (pp. 125-126). Castellón, España: Publicaciones de la Universidad Jaume I.

Romero, A., y Crisol, E. (2012). Las guías de aprendizaje autónomo como herramienta didáctica de apoyo a la docencia. Escuela Abierta, 15: 9-31. https:// repositorioinstitucional.ceu.es/handle/10637/7006 
Roque, Y., Valdivia, P., Zalagaz, M., y Alonso, S. (2017). Plan de acciones dirigido de auto aprendizaje en estudiantes de primer semestre de Enfermería. Educación Médica Superior. 31(4), http://ems.sld. cu/index.php/ems/article/view/1211/593

Salinas, M. I. (2011). Entornos Virtuales de Aprendizaje en la Escuela: tipos, modelo didáctico y rol del docente. http://www.uca.edu.ar

Salvador, F., y Gallego, J. L. (2009). Enfoque didáctico para la individualización. En: A. Medina y F. Salvador (Ed.) Didáctica General (pp. 247-272). Madrid, España: Pearson Educación.

Silva, H., R. Pinho, López, L., Nogueira, A., y Silveira, P. (2010). Illustrated plant identification keys: An interactive tool to learn botany. Computers and Education, 56: 969-973, DOI: 10.1016/j. compedu.2010.11.011.

Simpson, M. G. (2019). Plant systematics. San Diego, USA: Academic Press.
Sotelo, M., Echeverría, S., Ramos, D., y Barrera, L. (2015). Estrategias de aprendizaje utilizadas por estudiantes universitarios y su relación con el rendimiento académico. En R. Pizá, S. Mortis, M. González, y B. Orduño (Ed.). Resultados de Interacción Educativa (pp. 23-35). Obregón, México: ITSON.

Torres-Diaz, J. C., Jara, D. I., y Valdiviezo, V. (2013). Integración de redes sociales y entornos virtuales de aprendizaje. Revista de educación a distancia, 35: 1-22. https://revistas.um.es/red/article/ view/233651

Ulloa, L., y Velázquez, E. (2009). Un entorno virtual de enseñanza-aprendizaje con productos multimedia. Revista Cubana de Computación, 3: 48-51.

Velázquez, E. (2005). Estrategia didáctica para estimular el aprendizaje reflexivo en los estudiantes de las carreras de Ciencias Naturales en los Institutos Superiores Pedagógicos (tesis doctoral). Universidad de Ciencias Pedagógicas "Félix Varela", Santa Clara, Cuba. 


\section{Anexo 1. Diagnóstico inicial de los estudiantes del primer y segundo ańo de la carrera de Biología de la Universidad de Oriente.}

Estimado estudiante, bienvenido a la asignatura de Sistemática Vegetal. Te invitamos a leer atentamente la siguiente encuesta y a responder cada pregunta con sinceridad. Este cuestionario es anónimo y agradeceríamos tu participación y franqueza en la respuesta a las preguntas. El objetivo del conocer como organizas y realizas tu estudio para ayudarte a estudiar los contenidos de las asignaturas de Sistemática Vegetal en la carrera de Biología de la Universidad de Oriente.

Edad:

Sexo: femenino masculino

Curso:

1.- ¿Te gusta estudiar?

si

no

no se

2.- ¿Empleas alguna técnica de estudio?

si

no

no se

3.- Menciona cuál o cuáles técnicas de estudio conoces.

4. Selecciona en cuál o cuáles de los siguientes lugares prefieres estudiar:

Biblioteca En el cuarto Al aire libre

5.- Selecciona con quien prefieres estudiar:

Con nadie

Con los estudiantes sin problemas docentes

Con los estudiantes con problemas docentes

Con tus amistades

Con un grupo de estudiantes seleccionado por tu profesor

6.- Selecciona con qué frecuencia regularmente estudias

Diariamente

Tres veces a la semana

Semanalmente

Ante de las evaluaciones

Nunca

7.- ¿Qué tiempo dedicas regularmente al estudio?

Ningún tiempo

Menos de una hora

Entre 1 y 3 horas

Entre 3 y 5 horas

Mas de 5 horas

8.- Seleccione cuál o cuáles fuentes utilizas para estudiar

Internet Libros Notas de clase Guías de Estudio 
Anexo 2. Encuesta aplicada para medir la satisfacción de los estudiantes de la carrera de Biología de la Universidad de Oriente con el uso de las Guía de Estudio y Aulas Virtuales de Sistemática Vegetal.

Estimado estudiante, lee atentamente la siguiente encuesta a responder. Este cuestionario es anónimo y agradeceríamos tu participación y franqueza en la respuesta a las preguntas. El objetivo del mismo es validar la satisfacción del uso de las herramientas virtuales diseñadas para las asignaturas de Sistemática Vegetal en la carrera de Biología de la Universidad de Oriente.

Edad:

Sexo: __ femenino masculino

Curso:

1.- ¿Te gustan las Guías de Estudio de Sistemática Vegetal?
si
no
no se

2.- ¿Cuándo vas a estudiar las asignaturas de Sistemática Vegetal tu prefieres o no utilizar las guías de estudio?

si

no

no se

3.- ¿Cuáles son las secciones de las guías de estudio que más te gustan?

1

2

3

4.- ¿Cuáles son las secciones de las guías de estudio que menos te gustan?

1

2

3

5.- ¿Durante tu estudio utilizas las guías de estudio de las asignaturas?

si

no no se

6.- ¿Qué importancia usted les concede a las guías de estudio de Sistemática Vegetal?

7.- Si tuvieras la opción de utilizar o no las guías de estudio de Sistemática Vegetal durante tu auto preparación ¿la utilizarías?

si

no no se

8.- ¿Te gustan las actividades reflejadas en las guías de estudio? (MARQUE CON UNA X)

\begin{tabular}{|l|l|}
\hline Me gustan mucho & \\
\hline Me gustan más que lo que me disgustan & \\
\hline Me dan lo mismo & \\
\hline Me disgustan más que lo que me gustan & \\
\hline No me gustan nada & \\
\hline No se decir & \\
\hline
\end{tabular}


9.- ¿Qué aspectos a su juicio limitan el uso de las guías de estudio?

10.- ¿'Te gustan las Aulas Virtuales de Sistemática Vegetal? si no no se

11.- ¿Cuándo vas a estudiar las asignaturas de Sistemática Vegetal tu prefieres o no utilizar las Aulas Virtuales?

si no no se

12.- ¿Qué es lo que más te gusta de las Aulas Virtuales de Sistemática Vegetal?

1

2

3

13.- ¿Qué es lo que menos te gusta de las Aulas Virtuales de Sistemática Vegetal?

1

2

3

14.- ¿Durante las preparaciones para cursar las asignaturas de Sistemática Vegetal utilizas sus Aulas Virtuales?

si no no se

15.- ¿Qué importancia usted les concede a las Aulas Virtuales de Sistemática Vegetal?

16.- Si tuvieras la opción de utilizar o no las Aulas Virtuales de Sistemática Vegetal durante tu auto preparación ¿la utilizarías?

si no no se

17.- ¿Te gustan los contenidos de las Aulas Virtuales de Sistemática Vegetal? (MARQUE CON UNA X)

\begin{tabular}{|l|l|}
\hline Me gustan mucho & \\
\hline Me gustan más que lo que me disgustan & \\
\hline Me dan lo mismo & \\
\hline Me disgustan más que lo que me gustan & \\
\hline No me gustan nada & \\
\hline No se decir & \\
\hline
\end{tabular}

18.- ¿Qué aspectos a su juicio limitan el uso de las 